\title{
Pour un emboîtement médiatique et méthodologique
} Deux tableaux théoriques de Lorenzo Lotto

\section{Alessandro Rossi}

\section{(2) OpenEdition}

Journals

Édition électronique

URL : http://journals.openedition.org/imagesrevues/3108

DOI : 10.4000/imagesrevues.3108

ISSN : 1778-3801

Éditeur :

Centre d'Histoire et Théorie des Arts, Groupe d'Anthropologie Historique de l'Occident Médiéval, Laboratoire d'Anthropologie Sociale, UMR 8210 Anthropologie et Histoire des Mondes Antiques

\section{Référence électronique}

Alessandro Rossi, «Pour un emboittement médiatique et méthodologique », Images Re-vues [En ligne], 11 | 2013, mis en ligne le 14 janvier 2014, consulté le 30 janvier 2021. URL : http:// journals.openedition.org/imagesrevues/3108; DOI : https://doi.org/10.4000/imagesrevues.3108

Ce document a été généré automatiquement le 30 janvier 2021.

\section{(c) (i) (8)}

Images Re-vues est mise à disposition selon les termes de la Licence Creative Commons Attribution Pas d'Utilisation Commerciale 4.0 International. 


\section{Pour un emboîtement médiatique et méthodologique}

Deux tableaux théoriques de Lorenzo Lotto

Alessandro Rossi

«La distance temporelle n'est donc pas un obstacle à surmonter [...]. Il importe en réalité de voir dans la distance temporelle une possibilité positive et productive donnée à la compréhension. $»^{1}$ Hans-Georg Gadamer

Objets théoriques et Moyens visuels 
1 L'approche des tableaux de la Renaissance ou de l'époque moderne classifiés selon des catégories qui leur sont étrangères (simplement parce qu'elles sont conçues ou théoriquement articulées ultérieurement), peut sembler, pour celui qui possède une sensibilité historique, déplacée. Sur une telle approche plane le risque, ou la "faute", d'exposer l'objet artistique à une explication anachronique. Celle-ci revendique pourtant la possibilité critique de se détacher d'un positivisme historico-documentaire rigide, en le traversant sans s'attarder pour s'ouvrir à une pluralité et à une transversalité de

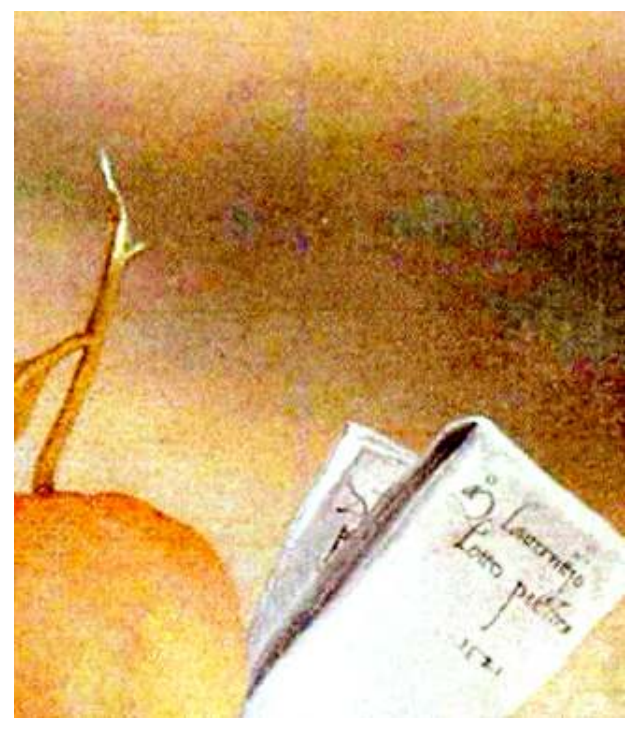
points de vue qui autorisent une compréhension plus claire des œuvres analysées ${ }^{2}$.

2 Il existe des tableaux qui, plus que d'autres, semblent ne pas être seulement la représentation de langage et pensée picturaux spécifiques mais coïncident exactement avec ceux-ci. Au-delà d'une lecture stylistique, iconographique ou iconologique, si l'on veut comprendre la structure qui articule de telles œuvres et les rend langage et pensée picturaux, il est nécessaire de traduire un tel langage dans la logique discursive qu'ils sous-tendent, en les rendant intelligibles, non pas seulement au regard sensible, mais aussi aux yeux de l'intellect, en remettant en cause cet acquis d'Heinrich Wölfflin selon lequel seuls les mots ont le pouvoir d'exprimer des concepts, alors que les images peuvent seulement s'occuper de l'aspect sensible et sentimental ${ }^{3}$. Que la peinture ait aussi une valeur discursive, et non pas seulement sensible, avait déjà été envisagé par Léonard de Vinci, qui (plus âgé que Lorenzo Lotto d'une génération) n'utilisa pas au hasard le terme «discorsi » (discours) pour désigner les valeurs picturales qui maintiennent et transmettent le message de l'artiste ${ }^{4}$.

3 À la suite des études d'Hubert Damisch, il est difficile d'avoir sur les œuvres d'art un regard qui n'essaie pas de reconnaître les caractéristiques paradigmatiques qui les marquent de façon intrinsèque ${ }^{5}$. Damisch se demande de quelle façon les objets deviennent modèles, paradigmes. Pour répondre à cette question, il définit une catégorie à la fois concrète et conceptuelle qu'il nomme objet théorique (theoretical object). Dans une conversation avec Yve-Alain Bois, Denis Hollier et Rosalind Krauss publiée en 1998 dans October, Damisch donne un bref aperçu de ce qu'il entend par là :

«Un objet théorique est quelque chose qui t'oblige à faire de la théorie et qui te fournit aussi les moyens pour la faire. Ainsi, si l'on accepte un objet dans des termes théoriques, celui-ci produira autour de lui des effets. En outre est objet théorique ce qui nous force à nous demander ce qu'est la théorie. Celui-ci se pose en termes théoriques ; il produit de la théorie ; et il nécessite une réflexion sur la théorie.» ${ }^{6}$

4 A la suite des réflexions de Damisch, nous pouvons dire qu'il existe des objets théoriques qui expriment à travers leurs possibilités visuelles ce qui est habituellement reçu comme théorie seulement verbale. Cette théorie se structure ainsi dans certaines œuvres peintes qui ne la disent pas mais la montrent simplement. La question reste donc, 
pour ceux qui veulent se rapprocher de ces objets théoriques, de les identifier et de les reconnaître, pour ensuite tenter de verbaliser la théorie qu'ils exhibent.

Comprendre cela permettrait d'analyser des tableaux anciens sans sous-estimer une valeur théorique qu'ils peuvent masquer en l'exposant, en la manifestant sur la surface de la toile, car elle coïncide avec le tableau lui-même. En d'autres termes, si l'analyse iconographique et iconologique permet de comprendre le sujet de l'œuvre et de la replacer dans son contexte socio-culturel - peut-être d'identifier la commande et une possible source littéraire -, le fait de pouvoir capter les dispositifs qui lui permettent de se placer sur le plan théorique de la représentation ne peut que passer par une spéculation sémiotique et philosophique qui traduise les signes visuels dans des énoncés théoriques réciproquement vérifiables

Fig. 1

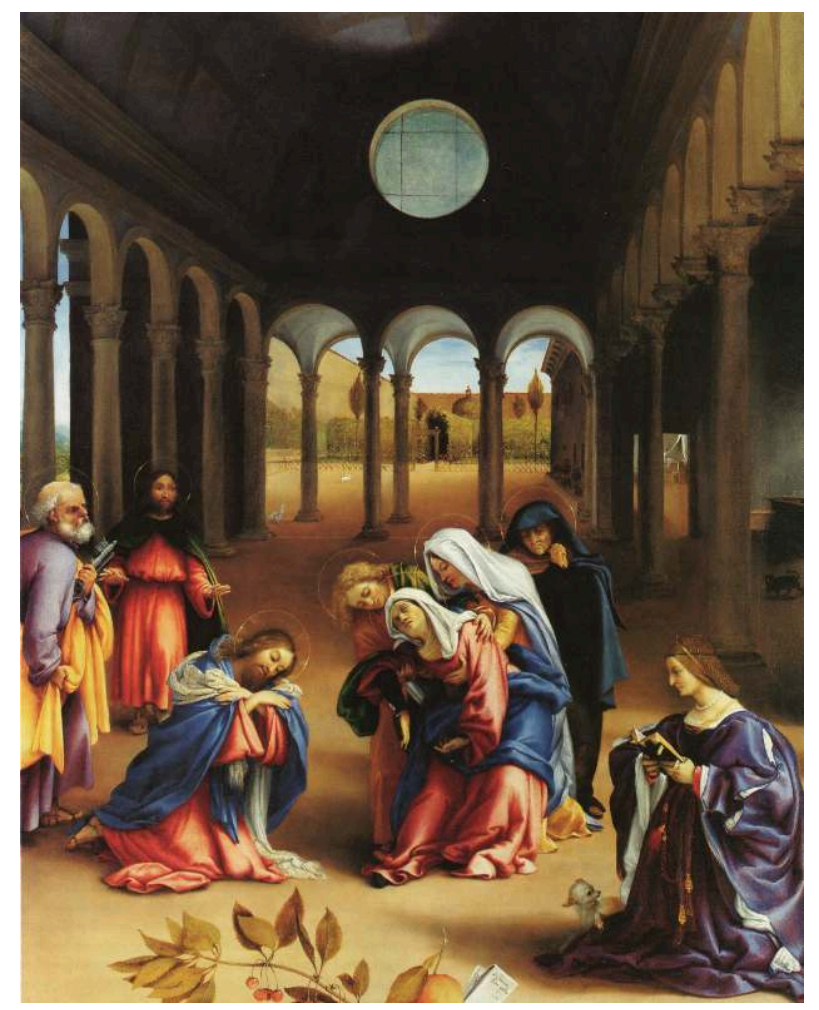

Lorenzo Lotto, Adieu du Christ à sa Mère, avec Elisabetta Rota, 1521, huile sur toile, 126 x 99 cm, Berlin, Gemäldegalerie

http://www.smb.museum/ 
Fig. 2

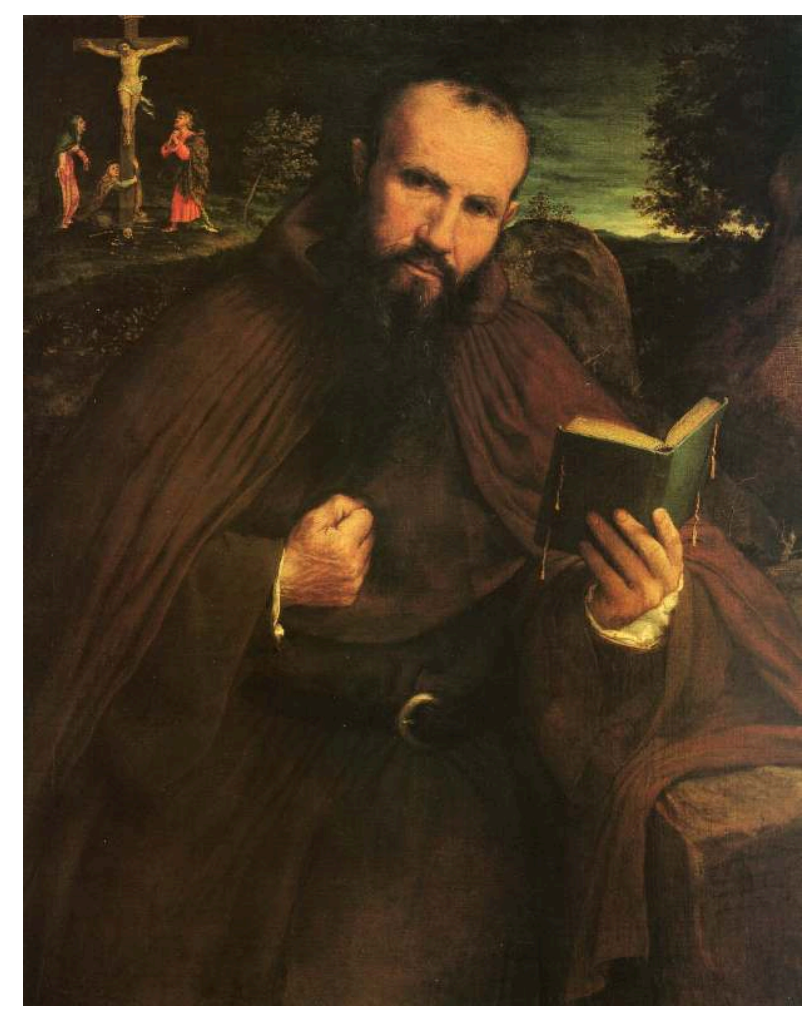

Lorenzo Lotto, Portrait de Fra Gregorio Belo, 1547, huile sur toile, 87,3 x 71,1 cm, New York, Metropolitan Museum

HTTP://WWW.METMUSEUM.ORg/

6 Le présent article identifie deux tableaux du peintre vénitien Lorenzo Lotto (1480-1556), l'Adieu du Christ à sa Mère, avec Elisabetta Rota (Berlin, Gemäldegalerie) [fig. 1] et le Portrait de Fra Gregorio Belo (New York, Metropolitan Museum) [fig. 2], peints respectivement en 1521 et en 1547, comme objets théoriques potentiels. Ces œuvres semblent effectivement structurées dans le but de montrer, en filigrane, une inévitable coexistence du mot, de l'image et de l'imagination. La coexistence de tels éléments a été, au niveau théorique, ré-élaborée par William J. Thomas Mitchell. Pour celui-ci en effet, il n'existe pas de médias exclusivement visuels car l'image picturale renvoie de toute façon à des images mentales par l'intermédiaire de la parole, et vice-versa. Mitchell articule sa théorie dans un article de 2005 au titre significatif, There are No Visual Media ${ }^{8}$, dans lequel il affirme (en synthétisant) que le médium verbal et le médium visuel, bien que différents, ne peuvent pas être perçus séparément. Ceux-ci se mélangent et se fondent dans la pensée de l'observateur et de l'auditeur ou du lecteur. La peinture, traditionnellement considérée par l'histoire de l'art comme médium d'abord optique ${ }^{9}$, ne l'est en réalité pas du tout ${ }^{10}$, car, justement, poursuit Mitchell, c'est la perception purement visuelle qui en premier lieu n'existe pas ${ }^{11}$. La pureté d'un médium, verbal ou visuel, est, comme le défend encore Mitchell, contredite par le terme même de moyen qui dans sa médiation implique un amalgame d'éléments sensoriels, perceptifs et sémiotiques ${ }^{12}$.

Observons donc avec attention les deux tableaux de Lorenzo Lotto pour comprendre comment la pensée et le langage pictural incarnés dans ces toiles et la "théorie des moyens» (Media theory) ici considérée, bien qu'absolument autonomes et 
historiquement indépendants, coïncident jusqu'à se superposer, possibles traductions l'un de l'autre.

8 Avant de passer à l'analyse des tableaux il est cependant nécessaire de mettre en évidence que la méthode que nous voulons appliquer ici n'est pas une sorte de posticonologie naïve où la recherche du basic-text (c'est à dire du texte littéraire qui souvent précède et informe l'image peinte) se transforme en recherche d'une confirmation théorique a posteriori de ce que le tableau semble suggérer. Le but de cet article n'est pas en effet de comprendre ce que ces tableaux représentent, c'est-à-dire leur sujet à partir d'un texte (étude qui a déjà été menée) ${ }^{13}$, mais de comprendre à travers deux exemples, de quelle façon et avec quelle efficacité la peinture peut s'exprimer par ellemême en exhibant des contenus théoriques intelligibles. Il s'agira par ailleurs de pointer les limites de l'identification et de l'approfondissement historique des sujets des deux tableaux de Lotto, qui contextualisent les composantes d'un dispositif théorique intrinsèque aux tableaux mêmes sans réussir cependant à en révéler la structure paradigmatique. C'est dire que l'iconographie de ces deux tableaux pose des questions auxquelles l'histoire ne peut répondre qu'avec l'accord de la théorie. Dans ce sens, ce que les deux tableaux représentent ne serait pas seulement le sujet représenté, leur titre, mais également (et plus profondément) l'exposition paradigmatique de l'impureté même de l'image. Déclaration évidente de l'inévitable mélange des médias entre eux dans la spécificité du médium pictural. Spécificité qui, comme le rappelle Mitchell, ne perd pas sa particularité, bien que tous les médias ne peuvent être que mixtes $^{14}$.

\section{Adieu du Christ à sa Mère, avec Elisabetta Rota}

9 Au sujet de l'Adieu du Christ à sa Mère, avec Elisabetta Rota il a été plusieurs fois suggéré que la scène sacrée qui domine la composition doit se comprendre comme une traduction visuelle de l'oraison mentale effectuée par Elisabetta Rota ${ }^{15}$. Francesca Cortesi Bosco a été la première à remarquer que la commanditaire - dont le portrait se retrouve dans la tableau - est également la spectatrice impliquée à l'intérieur d'une scène sacrée que son imagination même a convoquée par la technique de l'oraison mentale décrite dans certains livres dévotionnels de l'époque.

«Le drame qui se déroule devant Elisabetta Rota Tassi, dévote commanditaire du tableau, - écrit Cortesi Bosco en 1976 - semble évoqué par une méditation de la dame elle-même. Celle-ci, ayant suspendu la lecture de la Passion du Christ, et fixant son regard dans le vide, imagine le moment de l'adieu du Fils à sa Mère entre les murs domestiques, que l'on peut alors interpréter dans le tableau aussi bien comme la demeure du Christ que celle de Tassi. Le peintre n'associe donc pas la figure du commanditaire à l'image sacrée selon l'usage habituel, mais plutôt visualise une façon de prier selon la technique de l'oraison mentale. $»^{16}$

Oraison mentale qui est évidemment provoquée par le livre de prières que la figure agenouillée tient ouvert entre ses mains. Selon Cortesi Bosco, ce petit livre serait l'une des deux publications largement diffusées aussi en langue vulgaire, dans les premières décennies du XVIe siècle, où justement l'épisode de l'adieu du Christ devient un thème de méditation. Ces deux textes sont les «Devote meditationi sopra la passione del nostro Signore» (Florence, 1493 environ), version en langue vulgaire des «Meditationes de passione Christi » du Pseudo-Bonaventure et le «Zardino de oratione fructuoso $»(\text { Venise, } 1500 \text { environ })^{17}$. 
11 Lotto représente le moment où, après l'adieu prononcé par le Christ à sa mère, certains apôtres (Jean, qui soutient la Vierge, Pierre avec les clefs du Paradis et, selon les interprétations, Judas, Thomas ou Jacques le Majeur) ${ }^{18}$, accourent pour soutenir la lamentation et les pleurs de la Vierge accompagnée de deux femmes pieuses (probablement Marie Salomé, la plus âgée, et Marie, femme de Cleofas, la plus jeune) ${ }^{19}$. Bien que nous participions à la pathétique et dramatique description de la scène présentée dans le texte des «Devote meditationi ${ }^{20}$, la seconde référence semble pourtant plus appropriée, car au-delà de la mention de l'épisode, celle-ci suggère la technique pour le visualiser. Le passage que le « Zardino de oratione fructuoso » dédie à l'Adieu du Christ propose en effet au lecteur de s'imaginer devant les yeux de la pensée la forme et l'habit du Christ et les personnes qui sont en sa compagnie et qui conversent avec lui ${ }^{21}$. Nous assistons à la visualisation d'un épisode évangélique apocryphe qui s'est formé "nela mente» (dans l'esprit) de la commanditaire portraiturée et qui prend corps à côté d'elle. En outre, en exhortant le lecteur à avoir "chome uno specchio dananti dali occhi de la mente la vita sua $»^{22}$ ("comme un miroir devant les yeux de l'esprit de sa vie », celle du Christ), Nicolò da Osimo, probable auteur $\mathrm{du}$ « Zardino de oratione fructuoso $»^{23}$, introduit le thème du miroir et, avec celui-ci, le délicat concept d'espace hétérotopique exposé par le reflet qui, métaphoriquement, illustre de façon exemplaire la théorie selon laquelle la parole écrite (celle du livre) ne peut que se refléter dans l'imagination (celle d'Elisabetta Rota à l'intérieur du tableau) imagination qui, à son tour, se reflète dans l'image fixée par l'artiste et se fait peinture (le tableau même, aujourd'hui à Berlin).

12 Le choix de la part de la commanditaire de faire représenter la méditation-visualisation de l'épisode rare de l'Adieu du Christ à sa mère ${ }^{24}$ est probablement dû à la coïncidence, presque empathique, entre un moment difficile de sa propre vie et un double sens qui exprime cet épisode évangélique. La séparation du Christ de sa mère connote en effet le premier épisode dramatique de la Passion en même temps que la première annonce du salut, comme l'indiquent clairement les mots mêmes de Jésus dans les « Devote meditationi »: "Consolatevi adunche madre mia, perché l'afflictione e tribulatione mia sarà immenso gaudio alla generatione humana » ("Consolez-vous donc, ma mère, car ma souffrance et mes tribulations seront immense jouissance pour la génération humaine »). Elisabetta Rota aurait justement, en lisant et en méditant un épisode qui unit la douleur et son joyeux rachat ${ }^{26}$, trouvé un réconfort à l'angoisse causée par des menaces adressées, durant les années 1520-1521, à son mari et beau-frère Pietro Andrea par des bandits ayant déjà assassiné son autre beau-frère, Alvise Tassi, évêque de Recanati et Macerata. Pour cette raison, la commanditaire aurait à la fois voulu se faire représenter au sein de l'épisode évangélique et en même temps comme bâtisseur de celui-ci à travers son oraison mentale inspirée, très probablement, par la lecture de l'un des textes étudiés par Cortesi Bosco ${ }^{27}$.

Ce subtile croisement de réalité (portrait physique et psychologique de la commanditaire) et d'imagination (représentation de l'oraison mentale) se retrouve au niveau de la composition et démontre la complexité structurelle et la valeur théorique du tableau. À travers une formule picturale, Lotto montre la conscience que le tableau a de lui-même: la nature morte placée in limine entre l'espace de la représentation picturale et celui du spectateur, au-delà des possibles symbolismes religieux des fruits représentés ${ }^{28}$, porte cette fonction autoréférentielle. Le fait de se placer in limine entre espace réel et espace peint permet à la branche de cerises, à l'orange et au cartel avec la 
signature (qui constituent cette nature morte) de se relier, en tant qu'éléments d'un même système illusionniste, à la lunette optique développée par les colonnes entre lesquelles se déroule la scène (pseudo) évangélique. Ainsi toute la composition se structure selon une profondeur scandée par des plans perspectifs aussi bien optiques que mentaux. Si cette nature morte est la formule qui permet d'affirmer le tableau comme tel (c'est-à-dire comme représentation se différenciant de l'espace réel du spectateur), le portrait d'Elisabetta Rota Tassi, personnage historique, en se faisant elle aussi image à l'intérieur du tableau, déclare le passage de la réalité à sa représentation. Celle-ci se présente à son tour comme portrait qui tient un livre ouvert entre les mains, en introduisant l'idée de lecture, donc de méditation et d'oraison mentale, qui offre l'ultérieur passage à la visualisation de ce que cette figure est en train de lire et de méditer. Ce dispositif visuel à forte densité théorique, permet au spectateur de parcourir avec les yeux et l'esprit les différentes couches de l'œuvre peinte.

En traversant en effet l'espace de la représentation (significativement marqué par la signature du peintre même), le spectateur repère des figures qui, les unes à côté des autres comme à l'intérieur d'une coulisse de théâtre, représentent "celui qui imagine" et "celui qui est imaginé". Distinction précisément rendue possible à travers la représentation d'un livre ouvert entre les mains de l'un des personnages reconnu comme un portrait grâce à la forme de ses vêtements, aux bijoux qu'il porte et à sa coiffure.

\section{Portrait de Fra Gregorio Belo}

La même succession perspective et mentale scandée par le trio portrait-livre-vision est reproposée par Lotto en 1547 dans le Portrait de Fra Gregorio Belo. Portant l'habit de l'ordre auquel il appartient, celui des hiérosolymitains, le frère originaire de Vicence est représenté dans l'acte pénitentiel de se percuter la poitrine alors qu'il s'adresse intensément au spectateur. Dans la main gauche il tient un livre ouvert comme s'il voulait l'exhiber au spectateur. Dans son dos, sur le côté gauche, le Christ crucifié est représenté entre les dolents. Cette scène religieuse, comme celle qui figure sur le tableau de Berlin, a été reconnue plusieurs fois comme "représentation visionnaire " et comme "événement en train de se dérouler dans la pensée du personnage portraituré $»^{29}$. Sur le même axe diagonal, qui part de l'angle en bas à droite où sont inscrits sur une pierre carrée la date du tableau et des généralités sur Fra Gregorio ${ }^{30}$, s'alignent le livre ouvert (représentation du médium verbal), le regard intense du frère (d'où part l'imagination), et l'épisode de la Crucifixion avec les trois dolents (visionimage fruit de l'imagination). Comme pour le tableau de l'Adieu, plusieurs tentatives d'identification du livre ont été faites. Y est souvent reconnu une valeur exégétique significative pour la compréhension des deux œuvres. Dans le cas du Portrait de Fra Gregorio, le livre contient sur un quart de couverture une inscription qui, tracée en graphie abrégée, dit : « Homelie de Greg. ». Le nom abrégé « Greg. » pourrait se rapporter ou bien au frère Belo ou, plus probablement, à Grégoire le Grand ${ }^{31}$, auteur de l'« Omelie di Santo Gregorio Papa sopra li Evangeli » (probablement celui publié en huitième - $15 \mathrm{x}$ $11 \mathrm{~cm}$; 192 feuillets numérotés au recto - à Venise en 1543). N'ayant trouvé aucun sermon qui se rapporte expressément à la Crucifixion dans les homélies du Pape Grégoire, Ruggero Rugolo reconnaît dans le livre représenté une plausible copie du « Pio e christiano trattato detto Specchio di Croce " (écrit par le frère dominicain 
Domenico Cavalca au début du XIVe siècle, publié pour la première fois à la fin du XVe siècle et réimprimé chez Gabriel Giolito de'Ferrari en petit huitième - 10 x $7 \mathrm{~cm} ; 208$ feuillets numérotés sur le recto à Venise en $1543^{32}$ ). Dans ce texte, l'image de la Crucifixion est proposée comme motif de méditation à accomplir dans la solitude et le retrait spirituel «ne li boschi e ne le selve $»^{33}$ («dans les bois et dans les forêts »). Le milieu forestier de la scène, l'attitude de pénitence du frère, son appartenance à l'ordre des hiérosolymitains (adeptes du saint ermite Jérôme dont leur nom dérive), la présence de la Croix comme vision mystique du sacrifice du Christ - en accord avec des expressions comme : «Cristo è libro : nel quale è breviata la scrittura » («Christ est le livre : dans lequel l'écriture est abrégée ») ou encore "Cristo in Croce sta come libro aperto ( (Christ en Croix est comme le livre ouvert»), présentes dans le texte de Cavalca - ne peuvent que suggérer une certaine adhésion de l'image peinte par Lotto au texte en question ${ }^{34}$.

Sans rentrer dans les questions doctrinales qu'implique l'identification du livre tenu par Fra Gregorio ${ }^{35}$, nous voudrions seulement faire remarquer que tous les candidats retenus pour le tableau de Lotto ont en commun le concept d'introspection par la métaphore du miroir. Miroir dont le reflet, comme nous l'avons déjà vu dans le tableau de l'Adieu du Christ à sa Mère, au-delà de ses complexes implications théologiques, implique aussi, et nécessairement, le renvoi d'images intérieures (les scènes de la vie du Christ) à des images extérieures (la peinture qui la rend visible) à travers l'imagination (la méditation induite par le texte écrit). Dans le "Specchio di Croce » on lit en effet : " la croce è uno specchio nel quale si conosce ogni $\cos a »^{36}$ ( $«$ la croix est un miroir dans lequel on connait toute chose »), alors que dans le « Specchio interiore » de Battista da Crema, publié en 1540, autre livre candidat, d'après Rugolo, à pouvoir être reconnu comme celui que tient dans les mains Fra Gregorio Belo, on lit : «Bisogna che l'huomo apra bene li occhi suoi et spesso risguardi in questo specchio, se vuole vedere le macchie dell'anima sua $»^{37}$ ( « il faut que l'homme ouvre bien les yeux et regarde souvent dans ce miroir, s'il veut voir les taches de son âme »). Enfin, ce même Grégoire le Grand, auquel l'inscription du dos du livre se rapporte probablement, soutenait que les textes sacrés sont comme un miroir qui, placé devant les yeux de la pensée, permet de voir " interna nostra facies $»^{38}$. Ces observations sont également étendues au paysage par Peter Humfrey, pour qui son caractère sombre et orageux est à interpréter comme manifestation visuelle (ou plutôt comme reflet) du tumulte de la pénitence qui se joue dans l'âme du frère ${ }^{39}$.

À considérer ces deux tableaux de Lotto comme de potentiels objets théoriques, il faudrait alors analyser en termes théoriques, comme le suggère Damisch, la théorie que l'on en extrait (exposition de l'emboîtement médiatique) et réfléchir sur la théorie même, c'est-à-dire essayer d'énoncer les éventuelles conséquences épistémologiques qui peuvent dériver de cette théorie (emboitement méthodologique).

\section{Emboîtement médiatique (théorie visuelle - théorie verbale)}

L'analyse met en évidence une structure équivalente dans les deux œuvres. Il s'agit d'un dispositif constitué essentiellement de trois éléments qui, combinés savamment entre eux, deviennent en même temps les signifiés et les signifiants d'une théorie visuelle. Ces éléments sont: le portrait du commanditaire, le livre que la figure représentée tient ouvert entre les mains, et la représentation d'un épisode évangélique, 
qui apparaît devant le commanditaire portraituré (dans le cas d'Elisabetta Rota) ou derrière lui (dans le cas de Fra Gregorio Belo). Dans chacun des deux tableaux ces trois éléments se chargent, à l'intérieur du dispositif même dont ils constituent les lignes de forces ${ }^{40}$, de significations après-coup indissociablement liées qui donnent naissance à un enchaînement nécessaire de renvois. Autrement dit, les commanditaires portraiturés deviennent, à travers leur propre imagination, le relais des épisodes évangéliques, ces derniers étant le fruit de l'imagination déclenchée par la lecture du livre tenu ouvert dans les mains de la figure représentée. Ces épisodes lus passent, à leur tour, de l'imaginaire à l'image et manifestent une transition du livre au tableau. L'établissement d'une telle connexion au cœur de la représentation manifeste une conscience de ses conditions (surtout, comme nous l'avons vu, dans le tableau de l'Adieu). Les tableaux s'insèrent en tant que tels dans la dynamique de ce qu'ils représentent, dans le lien de la parole écrite, de l'imagination et de la vision, en surimposant le statut de l'image au dispositif. En d'autres termes, le tableau (aussi bien celui de Berlin que celui de New York) fait partie du dispositif même qu'il construit: en synthétisant sur la toile les visions mentales produites par la parole (écrite), il met en jeu une pensée-langage pictural et dévoile par là la nature paradigmatique des deux œuvres ici examinées. À ce stade de la réflexion, les deux tableaux ne peuvent en effet que se manifester comme objets théoriques, justement parce que la syntaxe visuelle par laquelle ils s'articulent suggère elle-même sa propre transcription en termes discursifs. Ils montrent, au-delà de leur iconographie spécifique, ce qu'a théorisé Mitchell, non seulement lorsqu'il a l'intuition que l'image picturale renvoie à des images mentales par le moyen de la parole, et vice-versa, mais surtout lorsqu'il revendique qu'un "médium apparaît à l'intérieur d'un autre comme son contenu, ou encore qu'il advient toujours un 'emboitement réciproque' (nesting) d'un médium à l'intérieur d'un autre ${ }^{41}$, et donc que le contenu d'un médium est toujours un autre médium $»^{42}$, exclut que parmi les différents médias qui se créent les uns dans les autres il puisse y avoir un rapport régulier et séquentiel de type historique et donc qu'il soit possible d'établir lequel apparaît en premier ou en dernier, justement parce que le phénomène de l'emboîtement réciproque comporte nécessairement la dissolution partielle d'un médium dans l'autre, leur amalgame spatial comme temporel.

Pour suivre jusqu'au bout cette idée, vérifier aussi bien la possible superposition des deux théories, celle, visuelle, exprimée par Lotto et celle, discursive, exprimée par Mitchell, que le caractère paradigmatique des deux tableaux exemplifiant une image peinte bâtie sur l'impureté médiatique qui la constitue, la demande des commanditaires (représentés dans chacune des toiles) d'une peinture qui reflète une imagination induite par la parole, est probablement passée elle aussi par l'image sommaire qu'ils pouvaient s'en faire et qu'ils auraient décrite au peintre. Par des mots à leur tour filtrés par l'imagination de l'artiste qui, en fixant sur la toile une image peinte, en a cristallisé la forme de façon définitive. Forme visuelle, le tableau, sera à son tour l'objet de descriptions verbales (ekphrasis) elles-mêmes évocatrices d'autres images mentales et d'autres mots, en engageant un cycle potentiellement infini que cet article, avec chacun de ses lecteurs, contribuera à perpétrer ${ }^{43}$.

Cette dernière considération semble déjà anticipée et comprise dans le tableau même de l'Adieu. La complexité structurale de l'œuvre est confirmée, dans le feuillet plié avec la signature de l'artiste, placé sur le seuil du tableau, par une lettre destinée à l'artiste lui-même (on lit en effet sur le feuillet : «M[agistr]o Laurenttjo Lotto pictor(i)», c'est-à- 
dire "au Maître Lorenzo Lotto peintre»). Une telle lettre (moyen verbal écrit par antonomase) aurait été envoyée, d'après Augusto Gentili, par la commanditaire Elisabetta Rota au peintre pour l'informer de la façon dont elle aurait voulu faire réaliser le tableau ${ }^{44}$ (la confirmation documentaire de ceci ne serait possible que si l'on retrouvait l'original de cette même lettre peinte). Lotto aurait placé ce message, non sans une certaine malice illusionniste, comme prologue et épilogue de la représentation en unissant, en un lieu symbolique, le seuil, deux moments distants entre eux: la commande passée à l'artiste et la signature de celui-ci sur le tableau accompli.

\section{Emboîtement méthodologique (Sémiotique visuelle - Iconologie)}

L'analyse conduite sur les deux tableaux de Lorenzo Lotto, considérés comme objets théoriques, place dans un rapport étroit histoire et théorie, contexte et concept. Comme l'affirme Damisch ${ }^{45}$, si la théorie ne peut faire abstraction de l'histoire, cette dernière ne peut non plus se libérer de la théorie : l'histoire nécessite une théorie gnoséologique qui l'encadre de façon critique ${ }^{46}$. Concevoir, comme cela a été fait ici, un tableau comme un dispositif constitué de lignes de force déterminées, ce n'est pas créer un échafaudage théorique, un exosquelette conceptuel au-dessus de la surface peinte, mais c'est, au contraire, dégager un châssis invisible qui rend l'image significative et efficace dans l'histoire. Cette approche, sémiotique ${ }^{47}$, ne se rapproche pas tant de la recherche iconologique qu'elle s'y emboîte elle-même. Dans l'Adieu du Christ à sa Mère, l'iconologue Augusto Gentili ne croit pas, par exemple, que le livre tenu par Elisabetta Rota puisse être les «Devote meditationi" ni le «Zardino de oratione fructuoso $»^{48}$. Position permise si elle n'était justifiée d'une façon qui laisse perplexe. Les observations de Gentili qui affirment et revendiquent la complexité iconologique de l'œuvre démontent en même temps les éléments de référence internes qui la justifieraient ${ }^{49}$. Affirmant que ce livret n'est pas un exemplaire des "Devote meditationi », ni d'autres publications récentes assimilées, Gentili écrit: "Del resto, sarà bene precisarlo, quel libretto non aiuterebbe la signora a evocarla e a visualizzarla, perché non la racconta $»^{50}$ (" D'autre part, il est important de le préciser, ce livret n'aiderait pas la dame à évoquer et visualiser [la scène], car il ne la raconte pas »). Gentili en mettant en discussion (de façon paradoxale et ironique) l'identité du livre mine en réalité l'une des lignes de force sur lesquelles se fonde le tableau. En effet la question ne se limite pas à comprendre quel livre spécifique, historiquement identifiable, la commanditaire tient dans les mains (iconologie), mais s'étend au problème de la logique structurelle de l'œuvre même (sémiotique visuelle), à la façon dont celle-ci a été conçue. Le livre établit la liaison cardinale entre l'image de la femme peinte et la vision qu'elle déclenche à travers la lecture. En niant qu'il puisse s'agir des « Devote meditationi » (ou d'un livre semblable), d'un écrit précisément réalisé pour favoriser les oraisons mentales, Gentili suggère que la Rota aurait demandé à Lotto (justement à travers la lettre expédiée au peintre, représentée dans le tableau) une iconographie complexe sans tenir entre ses mains l'un des textes identifiés par Cortesi Bosco. La commanditaire se serait fait portraiturée avec un quelconque livre de prières trouvé dans sa maison (presque au hasard, nous fait comprendre Gentili), qui ferait peut-être allusion aux contenus des sermons de Pietro da Lucca, écoutés probablement à Bergame avec son mari ou peut-être avec Lotto lui-même. Sermons pour lesquels, à la réalisation du tableau, Elisabetta Rota ne 
possédait pas de références écrites ou imprimées ${ }^{51}$. Cette interprétation impliquerait l'étrange situation qui verrait Elisabetta Rota se faire portraiturer dans une position où elle a entre les mains un livre qu'elle ne possédait probablement pas mais qui l'aurait conduite à visualiser un épisode (apocryphe) de la vie du Christ. En d'autres termes, si l'on suit le raisonnement de Gentili, la Rota aurait donc inventé, motivée par les sermons de Pietro da Lucca, l'utilisation d'un instrument capable de déclencher sa visualisation mentale. Ce qui nous importe n'est pas d'établir si Lotto a ou pas réellement peint un exemplaire des « Devote meditationi » ou du «Zardino de oratione fructuoso ", mais de souligner comment le livre est ouvert entre les mains de la commanditaire, assumant une valeur structurelle à l'intérieur de la composition ${ }^{52}$. En effet, pour quelle raison la Rota aurait-elle voulu se faire portraiturer ainsi (même s'il s'agit d'un livre quelconque trouvé dans la maison), sinon pour faire en sorte que cet élément soit inséré dans la complexe logique iconographique de l'œuvre? Comprendre cette exigence (de sa part, de celle de Lotto, ou des deux) fait sens si elle s'encadre dans la conscience qu'ont la commanditaire et le peintre que le livre représenté est un objet qui prédispose à l'oraison mentale d'un épisode évangélique (apocryphe ou non). Raisonnement qui évidemment vaut aussi pour l'autre exemple considéré, le Portrait de Fra Gregorio Belo. Le livre, dans chacun des tableaux, n'est pas un objet qui montre la simple dévotion des commanditaires. S'il est un peintre attentif, de façon presque maniaque, aux détails, et qui confère toujours à ceux-ci une signification précise, indispensable pour déchiffrer le sujet du tableau, c'est certainement Lorenzo Lotto. Cette attention aux détails est probablement réclamée par les clients eux-mêmes (les personnages portraiturés) pour que de tel objet précis, et pas un autre, puisse les représenter au mieux. La compréhension entre un peintre comme Lotto et ses commanditaires devait alors passer par une élaboration érudite, et probablement divertissante, de l'identité physique et spirituelle du portraituré que le tableau prend en charge $\mathrm{e}^{53}$. 
Fig. 3

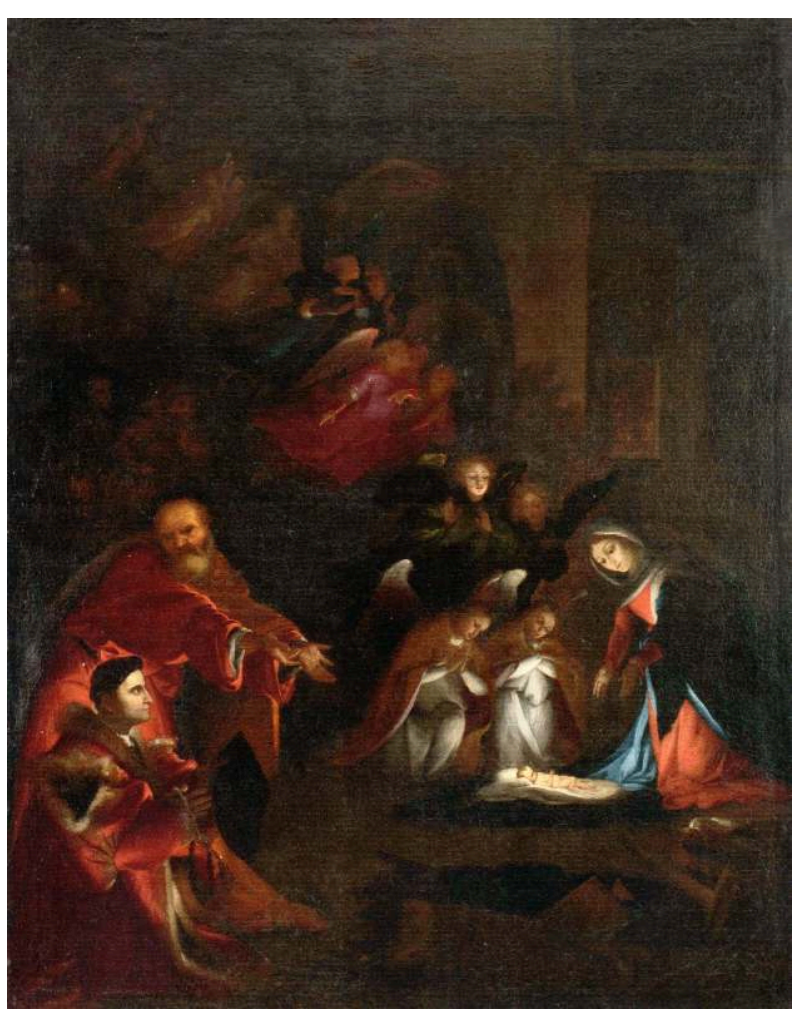

Peintre bergamasque d'après Lorenzo Lotto, Nativité adorée par Domenico Tassi (La Nuit), première moitié du XVIle siècle, huile sur toile, $132 \times 104$ cm, Venise, Gallerie dell'Accademia

http://www.gallerieaccademia.org/

Norberto Massi, sur la base de données documentaires, considère l'Adieu comme un pendant de la Nativité des Galeries de l'Académie à Venise [fig. 3] ; cette dernière s'en rapproche autant par son format que par la structure de sa composition ${ }^{54}$. (Malgré que ce tableau soit à considérer comme une copie mal conservée d'un original perdu ${ }^{55}$.) Dans ce tableau le commanditaire, Domenico Tassi (mari d'Elisabetta Rota), agenouillé et les mains jointes sur la gauche de la composition, est introduit par Saint Joseph, au moyen d'un geste éloquent, à la contemplation de l'Enfant nouveau-né et de la Mère en adoration. Comparée à la composition de l'Adieu, celle de la Nativité apparaît moins complexe, représentant une figure de la scène sacrée qui introduit "l'anachronique étranger" qui participe à l'événement. Bien qu'il soit vraisemblable que les deux toiles soient le fruit d'un projet dévotionnel unitaire conçu par les époux Tassi comme un double ex-voto, on peut se demander pour quelle raison ce pendant déséquilibre l'ensemble du point de vue de l'implication iconographique et de la complexité structurelle. Une réponse à cette question peut se trouver dans le fait que la Nativité de l'Académie n'est pas une œuvre de Lotto mais de l'un de ses copistes, en charge de reprendre du premier travail la composition générale mais en délaissant cette infinité de détails qui font le particulier des œuvres de Lotto, son épaisseur iconographique ${ }^{56}$. Il suffit d'évoquer, dans l'Adieu, ces éléments comme le chat qui saute dans l'ombre, les deux lapins blancs dans la cour, la nature morte au premier plan et le lit nuptial vide sur le fond, qui infusent l'ensemble de la composition de significations symboliques, bien qu'apparaissant d'abord comme des éléments décoratifs ou de pures bizarreries de l'artiste ${ }^{57}$. Nous pouvons présumer que la Nativité originale ne manquait probablement 
pas d'éléments iconographiques et structurels (lignes de force) à la hauteur de son pendant de Berlin ${ }^{58}$.

\section{Conclusion : deux tableaux paradigmatiques de Lotto}

La théorie de W. J. T. Mitchell nous a permis de reconnaitre dans deux tableaux spécifiques de Lorenzo Lotto l'inévitable création des médias qui advient dans chaque image, en identifiant le peintre comme un parfait interprète de cet emboîtement et les deux tableaux comme des œuvres théoriques paradigmatiques. À travers la gestion d'une intelligence picturale qui fait de l'image peinte une complexe et rigoureuse discipline de l'imagination, l'artiste vénitien lance ces deux toiles dans l'histoire, avec leur contenu théorique implicitement peint. De ce silence de la peinture (les tableaux disent mais ne parlent pas, ils sont muets avec éloquence) se "traduit", pour qui s'approche, ce qui du visuel pousse à la parole et transforme, sans solution de continuité, le langage des images en images du langage et vice-versa, dans la conscience que les deux moyens, dans leur propre spécificité, s'emboîtent l'un dans l'autre.

\section{NOTES}

1. Hans-Georg GADAMER, Vérité et méthode. Les grandes lignes d'une herméneutique philosophique, Paris, Éd. du Seuil, 1976, p. 137.

2. Nous comparons en ce sens et à titre d'exemples, deux articles récemment publiés sur deux revues françaises. Dans le premier cas une nouvelle perspective d'exégèse de l'œuvre picturale de Carlo Crivelli née d'une réflexion sur la distinction entre les concepts de filiation et alliance mise en œuvre par Gilles Deleuze et Félix Guattari dans Mille plateaux. Capitalisme et schizophrénie 2, Paris, Les Editions de Minuit, 1980 (Thomas GOLSENNE, « Génération Crivelli », Images Re-vues [En ligne], 9 | 2011, mis en ligne le 01 janvier 2011, consulté le 19 février 2012. URL: http:// imagesrevues.revues.org/1646). Dans le second cas, c'est la définition que Susan Sontag donne de «Camp» (« Notes on Camp», Partisan Review, XXXI, 1961, pp. 515-530) qui sert à approfondir l'imaginaire pictural de Gustave Moreau (Bijoy M. TRENTIN, « Gustave Moreau et les mythes : un regard Camp ", Art et Mythe. 20/21 siècles, sous la direction de F. Flahutez et T. Dufrêne, Nanterre, Presses Universitaires de Paris Ouest, pp. 37-46).

3. Heinrich WÖLFFLIN, Renaissance und Barock. Eine Untersuchung über Wesen Entstehung des Barockstils in Italien, München, Bruckmann, 1888. Consulté dans sa traduction italienne: Rinascimento e Barocco, Firenze, Vallecchi, 1928, p. 179.

4. Leonardo DA VINCI, «Il pittore ha dieci varii discorsi », dans Paola Barocchi, Scritti d'arte del Cinquecento, I, Milan-Naples, Ricciardi, 1971-1977, p. 477.

5. On pense tous au célèbre ouvrage Théorie du / nuage /. Pour une histoire de la peinture, Paris, Éd. du Seuil, 1972.

6. « A theoretical object is something that obliges one to do theory [...]. It's an object that obliges you to do theory but also furnishes you with the means of doing it. Thus, if you agree to accept it on theoretical terms, it will produce effects around itself [...]. It's theoretical object because it forces us to ask ourselves what theory is. It is posed in theoretical terms; it produces theory; and 
it necessitates a reflection on theory » (Yve-Alain BOIS, Denis HOLLER, Rosalind KRAUSS, Hubert DAMISCH, «A conversation with Hubert Damisch », October, 85, 1998, p. 8).

7. Sur la relation entre iconographie, iconologie et sémiotique visuelle voir: Umberto ECO, « Prospettive di una semiotica delle arti visive ", dans E. Mucci, P. L. Tazzi (sous la direction de), Teoria e pratiche della critica d'arte, Milan, Feltrinelli, 1979, pp. 69-70.

8. W. J. T. MITCHELL, « There Are No Visual Media », Journal of Visual Culture, 4, 2005, pp. 257-266.

9. "If we are looking for the best case of a purely visual medium, painting seems to be the obvious candidate. It is, after all, the central, canonical medium of art history. » (Ibid., p. 258).

10. "The painting we have habitually called 'purely optical', exemplifying a purely visual use of the medium, is anything but that. " (Ibid., p. 259).

11. "There are no purely visual media because there is no such thing as pure visual perception in the first place. » (Ibid., p. 264).

12. " The very notion of a medium and of a mediation already entails some mixture of sensory, perceptual and semiotic elements. » (Ibid., p. 260).

13. Francesca CORTESI BOSCO, «La letteratura religiosa devozionale e l'iconografia di alcuni dipinti di L. Lotto », Bergomvn, 1-2, 1976, pp. 12-14 ; Elizabeth NIGHTLINGER, « An iconographical study of Lorenzo Lotto's Christ taking leave of his Mother », in Source, vol. 14, n. 1, 1994, pp. $10-17$.

14. "The notion of 'medium specificity', then, is never derived from a singular, elemental essence. It is more like the specificity associated with recipes in cooking: many ingredients, combined in a specific order in specific proportions, mixed in particular ways and cooked at specific temperatures for a specific amount of time. In short, one can affirm that there are no 'visual media', that all media are mixed media, without losing the concept of medium specificity » (W. J. T. MITCHELL, « There Are No Visual Media », art. cit., pp. 260-261).

15. Lorenzo Lotto. Il genio inquieto del Rinascimento, catalogue de l'exposition, Washington, Gallery of Art, 2 novembre 1997 - 1 mars 1998, Bergame, Accademia Carrara di Belle Arti, 2 avril - 28 juin 1998, Paris, Galeries Nationales du Grand Palais, 12 octobre 1998 - 11 janvier 1999, sous la direction de D. A. Brown, P. Humfrey, M. Lucco, Milan, Skira, 1997, p. 123, Mauro LUCCO, fiche n. 17 ; Elise BOILLET CARAVAGLIOS, « Il congedo di Cristo dalla madre dipinto da Lorenzo Lotto e narrato da Pietro Aretino ", Venezia Cinquecento, XIII, 2003, 25, p. 99 ; Ottavia NICCOLI, Vedere con gli occhi del cuore. Alle origini del potere delle immagini, Rome-Bari, Laterza, 2011, pp. 90-94.

16. « Il dramma che si svolge dinnanzi a Elisabetta Rota Tassi, devota committente del quadro, sembra evocato da una meditazione della stessa gentildonna. La quale, sospesa la lettura della Passione di Cristo, fissando lo sguardo nel vuoto, immagina il momento del commiato del Figlio dalla Madre fra le pareti domestiche, nel dipinto interpretabili sia come dimora del Cristo che della Tassi. Il pittore dunque non accosta la figura del committente all'immagine sacra secondo l'uso consueto, bensì visualizza un modo di pregare secondo la tecnica dell'orazione mentale. » (F. CORTESI BOSCO, « La letteratura religiosa devozionale... ", art. cit., pp. 12-14).

17. Ibid.

18. Voir : E. NIGHTLINGER, «An iconographical study of Lorenzo Lotto... », art. cit., p. 12 ; Lorenzo Lotto..., catalogue de l'exposition, op. cit., p. 122 (avec bibliographie précédente) ; Augusto GENTILI, « Una lettera a Lorenzo Lotto (e altri dettagli) nel Congedo di Cristo alla Madre ", Venezia Cinquecento, XX, 2010 (2011), 39, p. 8.

19. Ibid., pp. 8-9.

20. Le texte est rapporté dans F. CORTESI BOSCO, « La letteratura religiosa devozionale... », art. cit., p. 12 .

21. Ibid., p. 14. Sur l'oraison mentale du «Zardino de oractione fructuoso » voir aussi : E. BOILLET CARAVAGLIOS, «Il congedo di Cristo dalla madre... », art. cit., p. 112.

22. F. CORTESI BOSCO, « La letteratura religiosa devozionale...», art. cit., p. 12.

23. Ibid., p. 11, note 21 . 
24. Francesco COLALUCCI, « Lorenzo Lotto, don Pietro da Lucca, Elisabetta Rota e il tema del Congedo di Cristo dalla Madre », Venezia Cinquecento, I, 1991, 1, pp. 27-60.

25. F. CORTESI BOSCO, « La letteratura religiosa devozionale », art. cit., p. 12.

26. Lorenzo Lotto..., catalogue de l'exposition, op. cit., p. 123.

27. F. CORTESI BOSCO, « La letteratura religiosa devozionale... », art. cit., pp. 12-14.

28. La nature morte a été mise en relation avec le sacrifice du Christ pour sauver l'humanité. Levi D'Ancona interprète l'orange comme le symbole du pêché à racheter et les cerises comme le symbole de la rédemption (Mirella LEVI D'ANCONA, The Garden of the Renaissance. Botanical Symbolism in Italian Painting, Florence, Olschki, 1977, p. 276). Cortesi Bosco associe les cerises aux joyaux du Paradis (F. CORTESI BOSCO, " La letteratura religiosa devozionale... », art. cit., p. 15). Gentili, en se détachant au contraire des précédentes interprétations, identifie la correspondance symbolique entre Marie, épouse du Christ, et l'orange alors qu'il renvoie le rouge des cerises au sang du sacrifice du Christ, en reliant ainsi les deux fruits aux valeurs symboliques de rédempteur et corédemptrice associées au Christ et à Marie (A. GENTILI, « Una lettera a Lorenzo Lotto... ", art. cit., p. 16).

29. Lorenzo Lotto..., catalogue de l'exposition, op. cit., Peter HUMFREY, fiche n. 50, p. 217 ; Ruggero RUGOLO, "Come un libro aperto. Lorenzo Lotto e Fra Gregorio Belo », dans Per il Cinquecento religioso italiano. Clero, cultura, società, Actes du Colloque Internationale d'Études, Sienne, 27-30 juin 2001, sous la direction de M. Sangalli, Rome, Edizioni dell'Ateneo, 2003, p. 212 ; O. NICCOLI, Vedere con gli occhi del cuore, op. cit., pp. 94-96.

30. «F. Gregorij belo de Vicentia / eremite in hieronymi Ordinis beati / fratris Petri de pisis. Anno / aetatis eius LV.MDXL-VII ». Inscription à relier aux nombreuses notes que Lotto écrit du 9 décembre 1546 au 11 octobre 1547 sur le Libro di spese diverse $(=c .52 \mathrm{v}$ et $=c$. $53 \mathrm{r}$ ). Dès la première note on peut, sans équivoque, identifier l'identité du commanditaire : "A dì 9 dicembre del 46, die dar frà Gregorio da Vicenza, dei frati de San Sebastian / da Venetia, per un suo retrato da naturale del qual fu fatto precio : / con un crocifissetto, la Madona, san Joanne e la Madalena, valse ducati 8" (Lorenzo LOTTO, Il "Libro di spese diverse" con aggiunta di lettere e altri documenti, P. Zampetti (éd.), Venise, Istituto per la Collaborazione Culturale, 1969, pp. 74-75).

31. Comparons : Maurizio GIMMARIOLI, "Fra Gregorio Belo », dans Il S. Girolamo di Lorenzo Lotto a Castel S. Angelo, catalogue de l'exposition, sous la direction de B. Contardi et A. Gentili, Rome, Società Editrice, 1983, pp. 112-113 et note 18; Massimo FIRPO, Artisti, gioiellieri, eretici. Il mondo di Lorenzo Lotto tra riforma e controriforma, Rome-Bari, Laterza, 2004, p. 287.

32. R. RUGOLO, « Come un libro aperto... », art. cit., pp. 208-209.

33. Ibid., p. 218.

34. Ibid.

35. Ces questions conduisent Rugolo à considérer l'inscription sur la quatrième de couverture "Homelie de Greg. » comme une couverture, ou plutôt comme une opération prudente de la part du commanditaire qui préféra, malgré les possibles équivoques que l'iconographie aurait pu provoquer, se faire portraiturer (toujours selon Rugolo) avec un livre écrit par un pape - saint qui plus est - et dans la plus stricte orthodoxie, plutôt qu'avec un texte qui aurait pu suggérer une position ambiguë du frère au sein de l'Eglise, comme pouvait être par exemple le «Specchio di Croce » ou encore davantage le «Specchio interiore » qui fut en effet mis au ban à peine huit ans après l'exécution du tableau (R. RUGOLO, « Come un libro aperto... », art. cit., p. 223).

36. Ibid., p. 222.

37. Ibid., p. 223.

38. Ibid., p. 221.

39. Lorenzo Lotto..., catalogue de l'exposition, op. cit., P. HUMFREY, fiche n. 50, p. 217.

40. C'est Gilles Deleuze qui affirme que chaque "dispositif comporte des lignes de forces». «Celles-ci - dit le philosophe dans sa dernière apparition publique à un congrès dédié à Michel Foucault - tirent des tangentes, enveloppent les trajets d'une ligne à l'autre, opèrent des va-et- 
vient du voir au dire et inversement, agissant comme des flèches qui ne cessent d'entrecroiser les choses et les mots, sans cesser d'en mener la bataille » (G. DELEUZE, «Qu'est-ce qu'un dispositif? ", in Michel Foucault philosophe, Rencontre internationale, Paris, 9-10-11 janvier 1988, Paris, Éd. du Seuil, 1989, p. 186).

41. "I would call 'nesting' a phenomenon in which one medium appears inside another as its content » (W. J. T. MITCHELL, "There Are No Visual Media », art. cit., p. 262). Que Mitchell considère comme typique phénomène d'emboîtement la télévision comme contenu du film (Ibid.) ne doit pas décourager l'application d'un tel concept à des moyens apparemment plus simples, comme la peinture.

42. Mitchell déclare de reprendre l'aphorisme de McLuhan : " the content of a medium is always an earlier medium » (Understanding Media, Cambridge, Ma, Mit Press, 1964) pour l'amplifier et l'éclairer (W. J. T. MITCHELL, « There Are No Visual Media », art. cit., p. 262).

43. Sur le rapport verbalisation - représentation il faut comparer: Cesare SEGRE, La pelle di San Bartolomeo. Discorso e tempo nell'arte, Turin, Einaudi, 2003 ; Pier Vincenzo MENGALDO, Tra due linguaggi. Arti figurative e critica, Turin, Bollati Boringhieri, 2005 ; Oscar SCHIAVONE, "Oltre la semiotica della pittura. Un pensiero pre-linguistico che si attua in parole e immagini », Ricerche di Storia dell'arte, 94, 2008, pp. 83-93.

44. A. GENTILI, « Una lettera a Lorenzo Lotto... », art. cit., p. 16 et note 18.

45. "I never pronounce the word theory without also saying the word history. Which is to say that for me such an object is always a theoretico-historical object. Yet if theory is produced within history, history can never completely cover theory. That is fundamental for me. The two terms go together but in the sense in which each escapes the other. » (Y. BOIS, D. HOLLIER, R. KRAUSS, H. DAMISCH, «A conversation with Hubert Damisch », art. cit., p. 8).

46. Sur ce thème on peut comparer le texte fondamental d'Henri-Irénée MARROU, De la connaissance historique, Paris, Éd. du Seuil, 1954.

47. Sur la sémiologie picturale on peut comparer: Louis MARIN, Études sémiologiques. Écritures, Peintures, Paris, Klincksieck, 1971.

48. A. GENTILI, « Una lettera a Lorenzo Lotto... », art. cit., p. 11.

49. Déplacer l'attention de l'œuvre en soi à l'analyse de ce qui a été dit sur celle-ci, comme si le discours sur l'œuvre était lui aussi englobé dans l'œuvre même, fait en partie référence à la sémiotique visuelle proposée par Jean-Louis SHEFER, Scénographie d’un tableau, Paris, Éd. du Seuil, coll. “Tel Quel”, 1969.

50. A. GENTILI, « Una lettera a Lorenzo Lotto... », art. cit., p. 11.

51. Même ne sachant pas l'effectif contenu de tels sermons, Gentili rappelle que le thème apocryphe de l'Adieu du Christ à sa Mère est traité dans un texte posthume de Pietro da Lucca intitulé Arte nuova del ben pensare e contemplare la Passione del nostro Signore Iesu Christo, publié à Bologne chez Girolamo Benedetti en 1523, deux ans donc après l'exécution du tableau de Lotto (A. GENTILI, « Una lettera a Lorenzo Lotto... », art. cit., pp. 7-8 ; Pour approfondir ce thème je renvoie à : F. COLALUCCI, « Lorenzo Lotto, don Pietro da Lucca... », art. cit.).

52. Du point de vue morphologique il peut être intéressant de remarquer comment le livre avec les mêmes renforts de protection du dos placés sur les quatre angles de la couverture et les fermoirs pour la fermeture sont représentés de manière quasiment identique dans les mains de Sainte Hélène dans la Sainte Conversation peinte en 1516-20 par Bonifacio de' Pitati, Madonna con Bambino e i santi Costantino, Elena e Giovannino (Florence, Palazzo Pitti, Galleria Palatina).

53. Gentili même, après avoir juré que le livre tenu dans les mains de la Rota n'était qu'un vieil objet de famille récupéré seulement pour la pose du tableau, revendique, en accord avec notre raisonnement, mais en contradiction avec celui-ci, que Lotto, comme chaque peintre, " représente en images la lecture qu'il a donnée et qui lui a été donnée d'un texte, à travers une exégèse visuelle personnelle ou partagée» («un pittore non traduce mai un testo ma rappresenta per immagini la lettura che ne ha data o che gli è stata data, attraverso una 
personale o condivisa esegesi visiva ») (A. GENTILI, « Una lettera a Lorenzo Lotto... », art. cit., p. 11). En affirmant cela le chercheur souligne l'importance de l'effective lecture d'un texte qui, pour avoir une exégèse visuelle, doit nécessairement avoir été réelle et spécifique et non pas inventée ou présumée.

54. Norberto MASSI, « Lorenzo Lotto's Nativity and Christ Taking Leave of His Mother : Pendant Devotional Paintings ", Artibus et Historiae, vol. 12, n. 23 (1991), pp. 103-109.

55. A. GENTILI, « Una lettera a Lorenzo Lotto... », art. cit., p. 7 et note 3. La récente restauration de l'œuvre a confirmé que la Nativité de l'Académie est sans aucun doute une copie, bien qu'ancienne, d'un original de Lotto (Omaggio a Lorenzo Lotto. I dipinti dell'Ermitage alle Gallerie dell'Accademia, catalogue de l'exposition, Venise, Galleria dell'Accademia, 24 novembre 2011 février 2012, sous la direction de R. Battaglia, M. Ceriana, Venise, Marsilio, 2011, M. CERIANA et G. HALLÉ, fiche n. 10, pp. 115-118).

56. Ces mêmes conclusions sont partagées par les auteurs de la fiche du catalogue de l'exposition où a été récemment exposée la Nativité en question (Omaggio a Lorenzo Lotto..., catalogue de l'exposition, op. cit., p. 118).

57. En guise de comparaison: E. NIGHTLINGER, « An iconographical study of Lorenzo Lotto... ", art. cit.

58. Boillet Caravaglios retient probable que dans ce diptyque Lotto ait voulu représenter deux types complémentaires de contemplation, une vision directe spirituelle et une vision rajoutée à travers la méditation (E. BOILLET CARAVAGLIOS, « Il congedo di Cristo dalla madre... », art. cit., p. 102).

\section{RÉSUMÉS}

Deux tableaux de Lorenzo Lotto, l'Adieu du Christ à sa Mère, avec Elisabetta Rota (1521) et le Portrait de Fra Gregorio Belo (1574) peuvent être interprétés en tant qu'objets théoriques selon la définition d'Hubert Damisch. Les structures de leur composition, comme leur iconographie, illustrent à leur manière la théorie développée par William J. Thomas Mitchell, selon laquelle le médium verbal et le médium visuel, au-delà de leurs différences, ne peuvent être perçus séparément. La méthode d'analyse suivie, qui utilise à la fois l'iconologie et la sémiotique visuelle, dans leur interdépendance, nous permet de voir dans ces deux tableaux un emboîtement mutuel des médias (verbal et visuel) au-delà des objets historiques spécifiques qu'ils représentent.

Two of Lorenzo Lotto's paintings, Christ Taking Leave of His Mother, with Elisabetta Rota (1521) and the Portrait of Fra Gregorio Belo (1547), are interpreted as theoretical objects according to the definition of this concept by Hubert Damisch. The compositional structure and iconography of these paintings illustrates independently William J. Thomas Mitchell's theory. According to this theory, the visual medium and the verbal medium cannot be separated, despite having clearly different characteristics. The method used here brings together Iconology and Visual Semiotics. This allows us to recognize the nesting media theory expressed in Lotto's two paintings, beyond the specific historical subjects they represent. 


\section{INDEX}

Mots-clés : Peinture, Lorenzo Lotto, objet théorique, Hubert Damisch, W. J. Thomas Mitchell, emboîtement réciproque

Keywords : Painting, Lorenzo Lotto, theoretical object, Hubert Damisch, W. J. Thomas Mitchell, nesting theory

\section{AUTEUR}

\section{ALESSANDRO ROSSI}

Alessandro Rossi a obtenu son doctorat Erasmus Mundus in Cultural Studies in Literary Interzones à l'Université de Bergame et de Perpignan. Il est spécialiste d'histoire de l'art italien du XVIe au XVIIIe siècle, d'iconologie, de sémiotique et d'anthropologie visuelle. Parmi ses plus récentes publications : «The Aristotelic 'phantasmata' \& the 'simulacra' theory of Lucretius in a painting attributed to Giorgione, Titian \& Sebatiano del Piombo ", dans Pratique du Hasard. Pour un matérialisme de la rencontre, sous la direction de J. Pollock, PUP, Perpignan, 2012, pp. 194-214 ; « Per il ciclo di Santa Caterina al Collegio dei Nobili di Parma. Documenti e note su Francesco Stringa » (avec Isabella Fogliani), Nuovi Studi, 17, 2011 (2012), pp. 129-140 ; « La pittura in cui abitano le ombre. Nota sulla pittura 'figurale' di Gianantonio Gennari », Elephant \& Castle [En ligne], 6, septembre 2012, URL : (http://cav.unibg.it/elephant_castle/web/numeri_monografici/ 1-ombra/12). 